\title{
Two cases of Pentalogy of Cantrell diagnosed antenatally at Korle Bu Teaching Hospital, Accra
}

\author{
Alim Swarray-Deen ${ }^{1}$, Joseph D. Seffah ${ }^{2}$ and Deliverance A. Antwi-Agyei ${ }^{1}$
}

Ghana Med J 2017; 51(4): 191-195 DOI: http://dx.doi.org/10.4314/gmj.v51i4.8

${ }^{1}$ Korle Bu Teaching Hospital - Obstetrics \& Gynaecology Department, Korle Bu, Accra, Ghana

${ }^{2}$ University of Ghana Medical School - Obstetrics \& Gynaecology Department, Accra, Ghana

Corresponding author: Dr. Alim Swarray-Deen

E-mail: asdeen8@gmail.com

Conflict of interest: None Declared

\section{SUMMARY}

Pentalogy of Cantrell is a rare congenital malformation syndrome that may be associated with other congenital anomalies. The syndrome is said to affect males and females in equal numbers and the prognosis is generally poor. We report two cases of Cantrell's Pentalogy diagnosed antenatally at the Korle Bu teaching Hospital. The 2 cases described in this report demonstrate the complete and incomplete variant of the syndrome. The cases were referred as an omphalocoele and a detailed anomaly scan confirmed the diagnosis of Pentalogy of Cantrell. Both cases were females and neither survived beyond 24 hours. Although this condition is associated with a poor prognosis, there have been some reported cases of survival in the literature. Thus, accurate prenatal diagnosis and a multidisciplinary approach to the management may offer improved outcomes.

Keywords: Foetal Anomaly, Ventral wall defect, Obstetric ultrasound, Prenatal diagnosis, Thoraco-abdominal syndrome

\section{INTRODUCTION}

Pentalogy of Cantrell is a rare congenital malformation syndrome first described in 1958 by Cantrell et al. ${ }^{1}$ It comprises of the following five characteristics:

- Ectopia cordis and intra-cardiac anomalies

- Lower sternal defect

- Midline supra-umbilical thoraco-abdominal wall defect

- Anterior diaphragmatic wall defect

- Defect of the diaphragmatic part of the pericardium. The estimated incidence of this rare syndrome is about 5.5 per 1 million live births. Incomplete forms of the syndrome with a combination of at least two defects have been reported in the literature. ${ }^{2}$ Both complete and partial forms have high mortality rates with prognosis depending primarily on the severity of the cardiac malformation.

We present 2 forms of the syndrome, which were diagnosed at the Korle-Bu Teaching Hospital in Ghana. In both cases, the mothers denied exposure to any form to teratogenic substances such as ionizing radiation, medications, alcohol, tobacco, cocaine, phenytoin, warfarin or valproic acid during the pregnancy.

There was no history of congenital malformations in the families.

\section{CASE REPORT 1}

The patient, a 37-year-old Gravida 3 Para 2, was referred from a peripheral hospital with a prenatal ultrasound diagnosis of an anterior abdominal wall defect (omphalocoele) made at 20 weeks of gestation. An initial first trimester scan at 10 weeks' gestation had demonstrated a midgut herniation suspicious of an omphalocoele (Figure 1).

A repeat ultrasound scan done at 26 weeks gestation, at our tertiary facility revealed a bulging structure arising from the anterior thoraco-abdominal wall that contained abdominal viscera (mainly the liver- $25 \mathrm{~m}^{2}$ ) and part of the heart (Figure 2).

The heart demonstrated a 4-chamber view with disproportion in ventricular sizes and a ventricular septal defect at the level of the crux cordis (the junction between the interatrial and interventricular septa).

The outflow tracts were not seen because of the ectopic position. In addition, bilateral talipes was identified. A prenatal diagnosis of Cantrell's pentalogy was made.

The parents were counselled regarding the diagnosis and associated poor prognosis, but decided to continue with the pregnancy. The neonatologists were consulted 


\section{Case Report}

as part of preparations for delivery and subsequent resuscitation. They suggested we allow vaginal delivery because of the expected poor prognosis. Pregnancy and delivery was closely supervised.

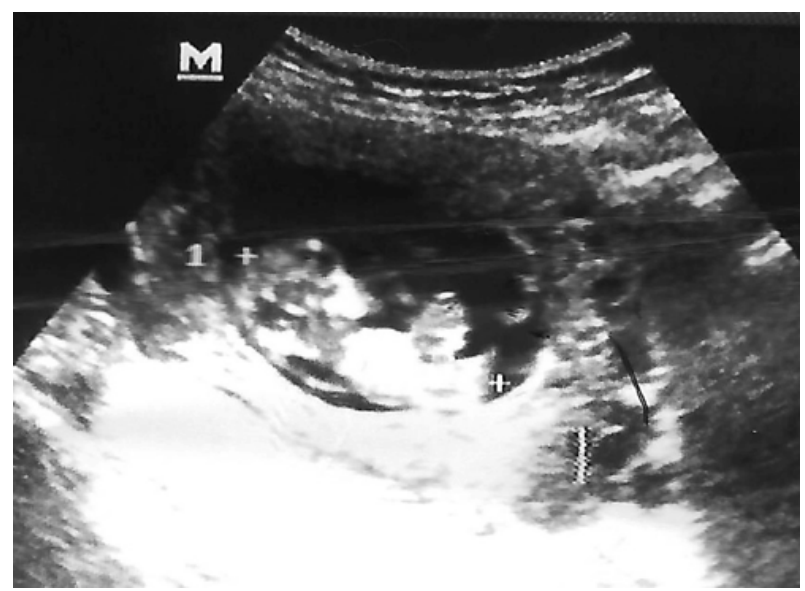

Figure 1 Scan at 10 weeks' gestation had demonstrating a midgut herniation suspicious of an omphalocoele

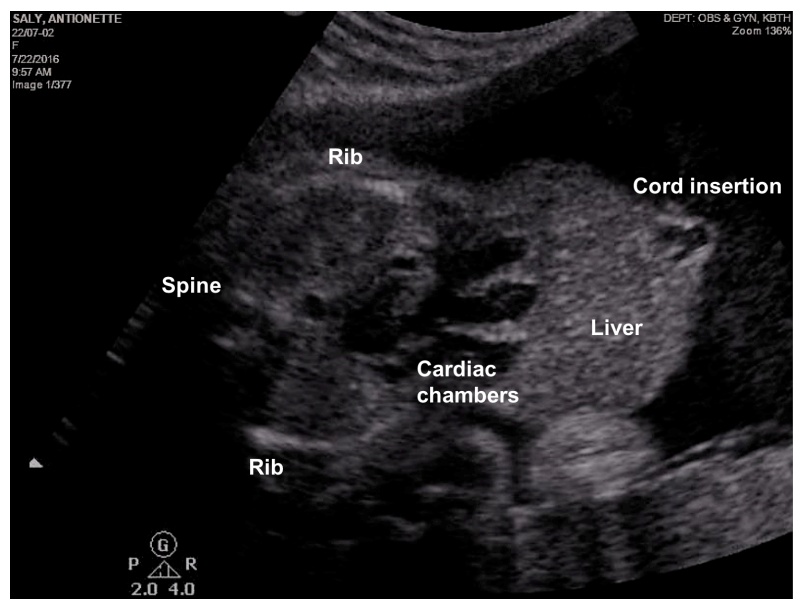

Figure 2 Ultrasound Scan at 26 weeks

The mother had a spontaneous vaginal delivery at 36 weeks of gestation. The baby was female and weighed $2.125 \mathrm{~kg}$, with Apgar scores of 4 and 5 at 1 and $5 \mathrm{~min}$, respectively. The baby had a length of $45 \mathrm{~cm}$ and occipito-frontal circumference of $35.8 \mathrm{~cm}$ which were appropriate for gestational age. The weight was below the $10^{\text {th }}$ centile for gestational age. Oxygen saturation was $60 \%$ on oxygen. The head and neck were normal and there were no dysmorphic facial features.

The baby was resuscitated at birth and transferred to the neonatal intensive care unit (NICU). Examination revealed a cyanosed baby in respiratory distress. There was an upper anterior wall midline defect with an omphalocoele and a pulsating mass above the omphalocoele, which was the apex of the heart (partial ectopia cordis). The defect was covered by a transparent membranous layer (Figure 3).

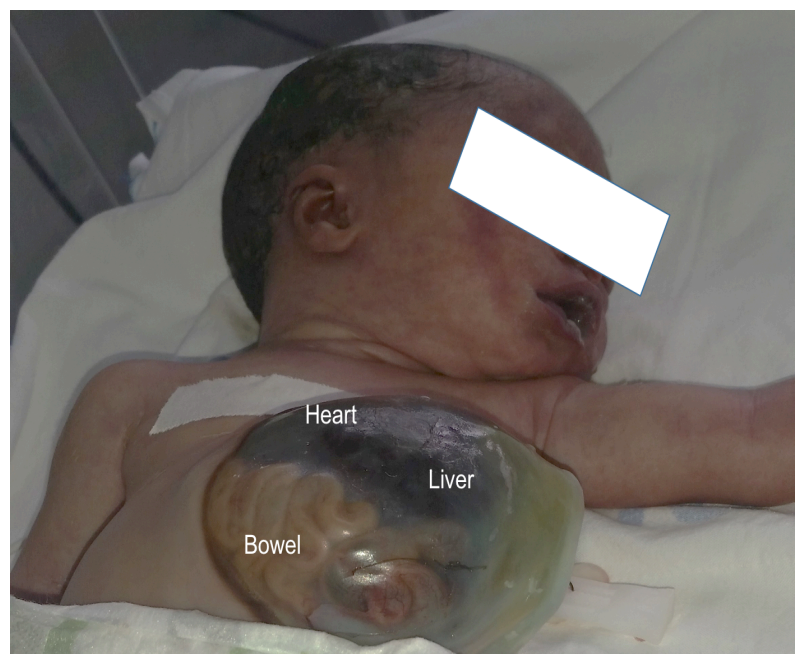

Figure 3 The defect covered by a transparent membranous layer

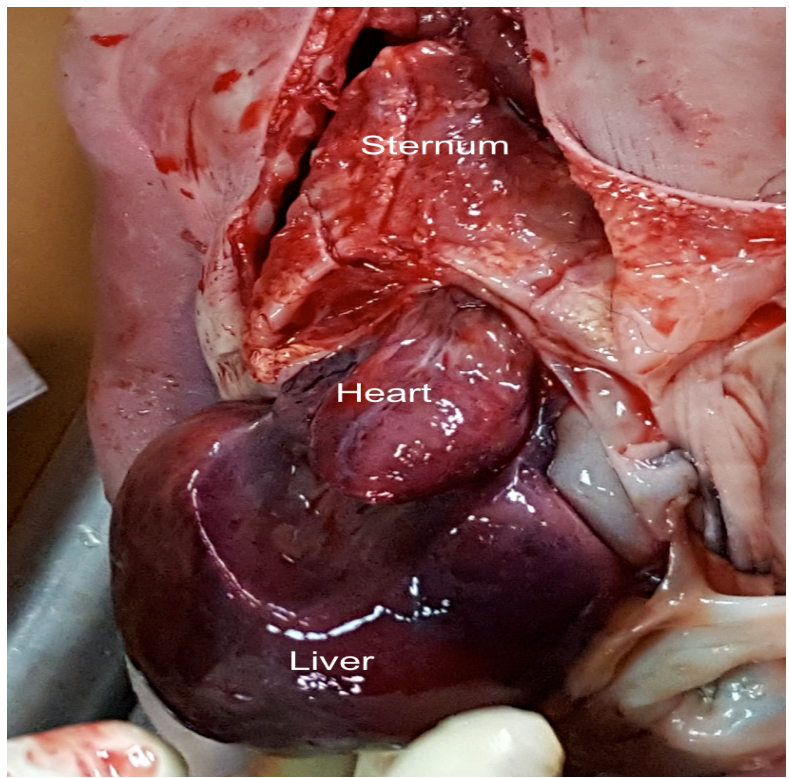

Figure 4 Showing The heart was located in the abdominal wall defect, lying freely on top of the liver

Palpation of the sternum showed an intact lower sternal wall. There was bilateral talipes equino-varus. No other defects were found on examination.

A diagnosis of incomplete pentalogy of Cantrell was made. Echocardiogram could not be done because the baby was in a critical condition and required resuscitation. The baby died two and half hours after admission at the Neonatal Intensive Care Unit (NICU). Post-mortem confirmed an anterior abdominal wall defect containing liver, heart and bowel. The sternum 
was complete and normal. There was no diaphragm over the liver but a posterior diaphragm over the kidneys. The liver was grossly enlarged with a groove on the upper surface accommodating the heart. The heart was located in the abdominal wall defect, lying freely on top of the liver (Figure 4). Further dissection revealed a Tetralogy of Fallot.

\section{CASE REPORT 2}

The patient was a 36-year-old, Gravida 3 Para 2, referred to our facility at term with an ultrasound diagnosis of an abdominal wall defect in the foetus. A repeat ultrasound showed an anterior abdominal wall defect with omphalocoele and a complete ectopia cordis. A diagnosis of pentalogy of Cantrell was queried.

Delivery was via the vaginal route as recommended by the obstetric and neonatal teams. A female neonate with birth weight of $3.394 \mathrm{~kg}$ was born at 38 weeks of gestation. Apgar scores were 4/10 and 5/10 at one and five minutes respectively.

Physical examination showed a cyanosed baby with the pulsating heart completely outside the chest at the level of the neck continuous with an exomphalos major $(>5 \mathrm{~cm})$. Oxygen saturation was $74 \%$ on oxygen. Lung fields were clinically clear. The sternum, the diaphragmatic portion of the pericardium and the anterior diaphragmatic wall were absent (Figure 5).

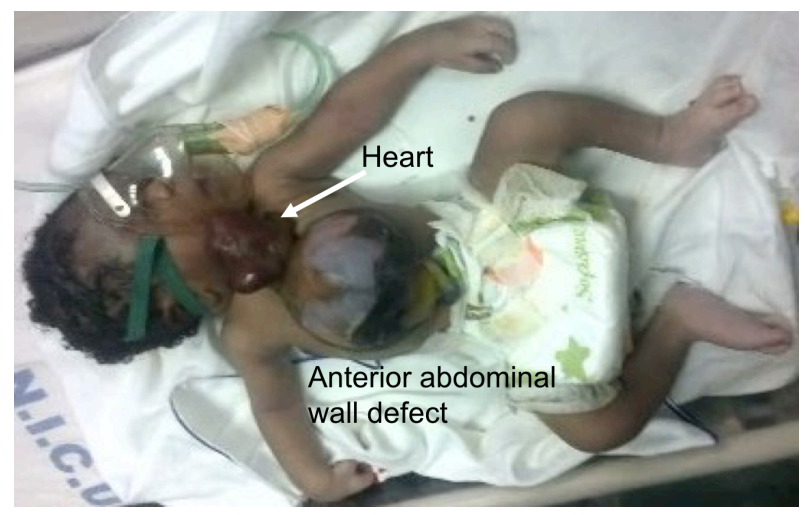

Figure 5 Complete Pentalogy of Cantrell

A diagnosis of complete pentalogy of Cantrell was confirmed. The baby died within 23 hours of delivery. The request for a post-mortem was declined by the parents.

\section{DISCUSSION}

Pentalogy of Cantrell (POC) occurs with varying degrees of severity, invariably causing severe, lifethreatening complications. ${ }^{3}$ Most infants do not have all the defects characteristic of the syndrome. The variability of the clinical components of the disorder from one individual to another can be significant. ${ }^{4}$

The syndrome affects males and females in equal numbers. Affected females have been reported to present with more severe symptoms. ${ }^{5}$ The two cases presented here were both female newborns. The exact prevalence is unknown but is estimated to be 1 per 65,000 to 100,000 live births. Error! Bookmark not defined., 8

The major hallmark of POC is an omphalocele associated with ectopia cordis. Various classifications have been used. The condition was previously simply classified as either Complete (full pentad) or Incomplete (at least two defects). ${ }^{1}$ Toyama in 1972 categorized the syndrome into three classes, wherein all the five defects were present in class I, four defects including ventral abdominal wall and intracardiac defects were present in class II, and a partial expression of this syndrome, always including a sternal defect was present in class III. $^{6}$

This condition can also be classified into 4 types based on the position of the heart. In cervical ectopia cordis $(3 \%)$, the heart is displaced superiorly into the area of the neck. In the thoracic variety of ectopia cordis $(60 \%)$, the heart protrudes anteriorly through a sternal defect. In the thoraco-abdominal variety of ectopia cordis $(7 \%)$, the heart is displaced outside the chest through a defect in the lower sternum in association with diaphragmatic and ventral abdominal wall defects. In the abdominal variety of ectopia cordis $(30 \%)$, the heart is displaced inferiorly into the abdomen through a defect in the diaphragm. ${ }^{7}$

From the cases presented, Case 1 depicted a Class II (incomplete) with an abdominal variety of ectopia cordis. Case 2 comparatively was a Class 1 (complete) with cervical ectopia cordis.

The exact cause of pentalogy of Cantrell is unknown. One theory suggests that it is due to an abnormality in the development of midline embryonic tissue 14 to 18 days post- conception. ${ }^{8}$ Failure of development of the septum transversum is responsible for the diaphragmatic and pericardial defects whereas the sternal and abdominal wall defects are believed to be due to impaired migration of mesodermal structures. ${ }^{9}$ Some familial cases have been reported indicating that genetic factors may play a role in the development of the disorder. ${ }^{\text {Error! Bookmark not defined. }}$ 
Antenatally, the diagnosis is made by ultrasound, usually after the first trimester because bowel herniation out of the abdomen is a normal event in foetal development before twelve weeks. ${ }^{10}$ As seen in Case 1, it is extremely difficult distinguishing a midline gut herniation from an anterior midline defect in the first trimester. There may be a potential role for the use of maternal serum markers such as alpha foeto-protein (AFP) and acetylcholinesterase which are elevated in cases of anterior abdominal wall defects. ${ }^{11}$ Results of ultra-sonographic studies suggest that in differentiating between normal physiologic herniation and an omphalocoele, one should note that physiologic midgut herniation should not exceed $7 \mathrm{~mm}$ in diameter. ${ }^{12}$ Other diagnostic methods such as CT scan and MRI can be used for confirmation. Error! Bookmark not defined.

The prognosis of Cantrell's pentalogy depends on the severity of intra and extra cardiac defects, as well as the extent of abdominal wall defect and diaphragmatic herniation. Associated intra-cardiac anomalies may include ventricular and atrial septal defects, Ebstein's anomaly, truncus arteriosus, transposition of great vessels, tetralogy of Fallot, and hypoplastic left heart syndrome. ${ }^{13}$ Those with an incomplete form had a better prognosis and/or survival than those with the complete form. The mean reported survival without any interventional surgery is about 36 hours. ${ }^{8}$ Both cases presented in this series died before 24 hours of age and received no surgical intervention.

The baby with the incomplete form of the disorder died earlier than the infant with the complete form. Unfortunately, neither echocardiogram nor post-mortem could be conducted for the baby in case 2 to confirm or rule out any intra cardiac defects. Hence we cannot be certain about the reasons for the differences in their survival time. Studies have shown that even with multiple corrective surgeries and intensive monitoring, morbidity and mortality rates are high and the long term prognosis is poor. ${ }^{8}$

Despite the rarity of the syndrome, the two cases presented were diagnosed antenatally within a period of one year. Out of the 30,000 antenatal cases seen within the year, the two cases reported were seen within the same month. This may be a sporadic occurrence. However, it may also indicate that this condition is not as rare as reported in the literature especially when an autopsy is not routinely offered for perinatal deaths. Various cases with different combinations of the defects have been reported in the literature including a number of cases reported in the sub-region. ${ }^{14,15}$
In our opinion, without the anomaly scan, the antenatal diagnosis would likely have been missed entirely or categorized simply as an omphalocoele. The role of detailed anomaly scans cannot be over-emphasized. Many more of such cases may be diagnosed antenatally and the affected women counselled accordingly. Anomaly scans will also help determine the true incidence of the syndrome in our sub-region. A multidisciplinary approach involving the neonatologist and paediatric surgeons is needed to achieve the best possible outcome for such babies.

\section{ACKNOWLEDGEMENT}

We wish to acknowledge the contributions of the staff of the Department of Child Health and Pathology of Korle Bu Teaching Hospital.

\section{REFERENCES}

1. Cantrell JR, Haller JA, Ravitch MM. A syndrome of congenital defects involving the abdominal wall, sternum, diaphragm, pericardium and heart. Surg Gynecol Obstet 1958; 107: 602-4

2. Desselle C, Herve P, Toutain A, Lardy H, Sembely C, Perrotin F. Pentalogy of Cantrell: sonographic assessment. J. Clin Ultrasound. 2007; 35(4): 216220

3. Pentalogy of Cantrell - NORD (National Organization for Rare Disorders). (n.d.). Retrieved February 7, 2017, from https://rarediseases.org/rarediseases/pentalogy-of-cantrell/

4. Hornberger LK, Colan SD, Lock JE, Wessel DL, Mayer JE Jr. Outcome of patients with ectopia cordis and significant intracardiac defects. Circulation. 1996; 1; 94(9 Suppl):II32-37

5. Zidere V, Allam LD. Changing findings in pentalogy of Cantrell in fetal life. Ultrasound Obstet Gynecol. 2008;32:835-837.

6. Toyama W.M. Combined congenital defects of the anterior abdominal wall, sternum, diaphragm, pericardium, and heart: a case report and review of the syndrome. Pediatrics. 1972 50:778-792

7. Sepulveda W, Wong AE, Simonetti L, et al. Ectopia cordis in a first-trimester sonographic screening program for aneuploidy. $J$ Ultrasound Med. 2013;32(5):865-71

8. Jafarian AH, Omidi AA, Fazel A, Sadeghian H, Joushan B. Pentalogy of Cantrell: a case report. $J$ Res Med Sci. 2011;16:105-109.

9. Morales JM, Patel SG, Duff JA, Villareal RL, Simpson JW. Ectopia cordis and other midline defects. Ann Thorac Surg. 2000;70:111-4

10. Varras M, Anastasiadis A, Akrivis C, Gialelis J. Pentalogy of Cantrell in the human foetuses: A rare congenital malformation. OA Case Reports 2013; $15 ; 2(15): 143$. 


\section{Case Report}

11. Calzolari E, Bianchi F, Dolk H, Milan M. Omphalocoele and gastroschisis in Europe: a survey of 3 million births 1980-1990. EUROCAT Working Group. Am J Med Genet. 1995; 58:187-94

12. Omphalocele Imaging: Overview, Magnetic Resonance Imaging, Ultrasonography. (n.d.). Retrieved February 7, 2017, from http://emedicine.medscape.com/article/404182overview\#showall
13. Patil AR, Praveen LS, Ambica V. Pentalogy of cantrell: A case report. BJR Case Rep. 2015;201520140002.

14. Shwe DD, Toma B. B, Ogbu, O, B, Shitta A. B. Complete Pentalogy of Cantrell: A case report and a review of literature $\backslash n$. IOSR Journal of Dental and Medical Sciences (IOSR-JDMS), 2014; 13, 01-04.

15. Okafor HU, Oguonu T, Uwaezoke SN, Anusiuba BC. A variant of pentalogy of cantrell in a live birth. Case Report. 2011; 14(1) : 106 - 108. 